\title{
A Cross-Sectional Study Examining How Patients in an Urban Practice Would Choose Between Regimen Options in Adjuvant Breast Radiotherapy
}

\author{
Jia-Xin Tracy Wang*1, Koh Wee Yao ${ }^{1}$, Choo Bok Ai ${ }^{1}$, Arul Earnest ${ }^{2}$, Jeremy Tey Chee Seong ${ }^{1}$ and Keith Lim Hsiu \\ Chin $^{1}$ \\ 1Department of Radiation Oncology, National University Cancer Institute, Singapore
}

2Department of Epidemiology and Preventive Medicine, Monash University, Australia

Submission: March 27, 2017; Published: April 05, 2017

"Correspondence Address: Jia-Xin Tracy Wang, Department of Radiation Oncology, National University Cancer Institute, Singapore (NCIS), 1 Kent Ridge Road, Singapore 119082, Tel: +65-6779-5555; Mob: +65-8125-8358; Email: tracy_jx_wang@nuhs.edu.sg

Abstract

Introduction: Randomised trials show equivalent rates of survival, local control and late toxicity with hypofractionationed breast radiotherapy (HBRT) and standard fractionation (SBRT). Reviewing patients' preferences given evolving evidence and patients' other considerations would inform our understanding of determinants in treatment choice. It would also guide the planning of radiotherapy resource usage. This study's aim was to determine patients' choice of schedule and reasons.

Methods: Using a decision board, advantages and disadvantages of adjuvant radiotherapy with 42.56 Gray in 16 fractions, and 50 Gray in 25 fractions were discussed with patients with T1-T2 N0 M0 breast cancer. Patients were asked to choose a fractionation schedule and provide their reasons. This was correlated with patient demographics.

Results: 74 patients were recruited of which $48.7 \%$ chose HBRT. Patients $50-60$ years were 5.42 times (95\% CI 1.72 to 17.02 , $p=0.004$ ) and patients 60-70 yrs were 10.59 times ( $95 \%$ CI 2.4 to $46.75, p=0.002$ ) more likely to choose HBRT than patients less than 50 yrs. HBRT was chosen for increased convenience (97\%) and lower cost (42\%) whilst SBRT for the reason of longer follow up (97\%).

Conclusion: Older patients chose HBRT for the advantage of cost and convenience, while younger patients chose SBRT because of longer length of clinical experience and follow-up. Research into patient preferences for breast radiotherapy would inform management of patient care and inform healthcare policy and resource allocation.

Keywords: Breast neoplasms; Decision making; Shared; Dose fractionation; Patient preference; Radiotherapy; Adjuvant

Abbreviations: HBRT: Hypofractionationed Breast Radio-Therapy; SBRT: Standard fractionation Breast Radio-Therapy; ECOG: Eastern Cooperative Oncology Group

\section{Introduction}

Early stage breast cancer treated with breast conservation surgery presents a unique situation where various options for adjuvant radiotherapy regimens exist and are well-studied with competing outcomes in efficacy and toxicity. A plethora of data is available and more will be contributed to the literature. Existing data is open to interpretation and physicians and national health policy disagree on the optimal choice of treatment. Furthermore, the patient population considering this treatment traverses the spectrum of ages and has competing considerations apart from the medical data [1-5]. The discussion with the patient on the choice of treatment is necessarily nuanced as is the patient's decision-making process.
Since the conduct of this study, publications presenting data on the experience of acute toxicities of adjuvant breast radiation therapy suggest hypo fractionation to have improved outcomes $[6,7]$. Only two randomized trials have published long-term follow-up data demonstrating equivalence in local control and late effects (long term cosmesis) for hypo fractionated adjuvant radiation therapy for early stage breast cancer $[8,9]$.

While patients were recruited for this study (from November 2009 to March 2010), other than results from Whelan et al. [8], the other randomized trials had only a median follow up approximating 5 years. Despite the relatively short follow up period in a disease with a long natural history, the medical 
community has been accepting of the use of HBRT. Its use is advocated in many national guidelines with the clinical consideration that any potential benefit from the longer schedules with higher total dose may be offset by the lower cost and increased convenience for patients who chose HBRT. Since the time of this study, the publication of updated START B [9] data suggests hypo fractionated regimens with lower doses may have improved late cosmetic outcomes and equivalent survival compared with standard fractionation RT.

A patient's ultimate choice of therapy would be contributed to by a myriad of factors including:

a) Data to date establishing equivalent outcomes for survival over durations studied,

b) START B data demonstrating a possible benefit for cosmesis with HBRT,

c) physician interpretation of emerging data for efficacy, toxicity outcomes from studies of adjuvant radiotherapy in breast cancer as well as toxicity data from studies of other tumour sites that share the same organs at risk,

d) how information is summarized and conveyed to the patient in the setting of a consult,

e) physician's professional experience, preferences and biases,

f) non-medical factors considered by the patient such as convenience, personal values, and perception of acceptable risk

g) pertinent remuneration and health economics policies

Certain technical and treatment-related factors such as breast size, would also factor into whether a particular patient would be offered a specific radiation therapy regimen. Larger breast size would give rise to prohibitively greater dose in homogeneity which may render hypo fractionated regimens impracticable due to the principle of 'double trouble' and its effect on late toxicities. Looking forward, where there is 15 year data for SBRT outcomes [10], longer term data from hypo fractionation trials is awaited.
Given all of these reasons, it is not surprising that differences in fractionation preferences between centres have been well documented [11-13]. When presented with a choice, patients may wish to have longer fractionations even with only a small potential benefit $[14,15]$. Studies have also demonstrated that a difference exists between what oncologists consider important and patients' beliefs, as well as physicians' understanding and perception of patients' beliefs $[16,17]$. Patients have also indicated a wish to have more information about their disease and a desire for shared decision [18-20]. This is particularly true for patients with breast cancer.

Given the multiple uncertainties in predicting a patient's final decision for adjuvant breast radiation therapy, we proposed using a decision board instrument based on the results of the Canadian breast cancer study to determine which fractionation schedule patients would choose [21]. Prior studies using decision boards have demonstrated that they are feasible and acceptable to both patients and doctors, resulting in improved communication and facilitating shared decision making [21,22]. Decision boards have been shown to be well understood and easily implemented in non-English speaking populations as well [23].

\section{Methods}

The primary end point of this study was to determine what percentage of patients of our urban practice diagnosed with T1 - T2 N0 M0 breast cancer, following breast conservation surgery would choose HBRT over SBRT. Secondary end points were to elucidate the patient variables that influence this decision. In order to investigate these questions, a decision board based on the results from the keynote study by Whelan et al, was used (Table 1) [8]. The board presented a summary of the similarities and differences of two options of adjuvant breast radiotherapy regimens: 50 Gray in 25 fractions and 42.56 Gray in 16 fractions in terms of local control, cosmetic outcome, late toxicity and length of follow up. Factors such as cost and number of visits were also shown.

\section{Decision Board Used To Counsel Patients on Hypo- fractionation}

Table 1: The differences and similarities between the 2 treatments.

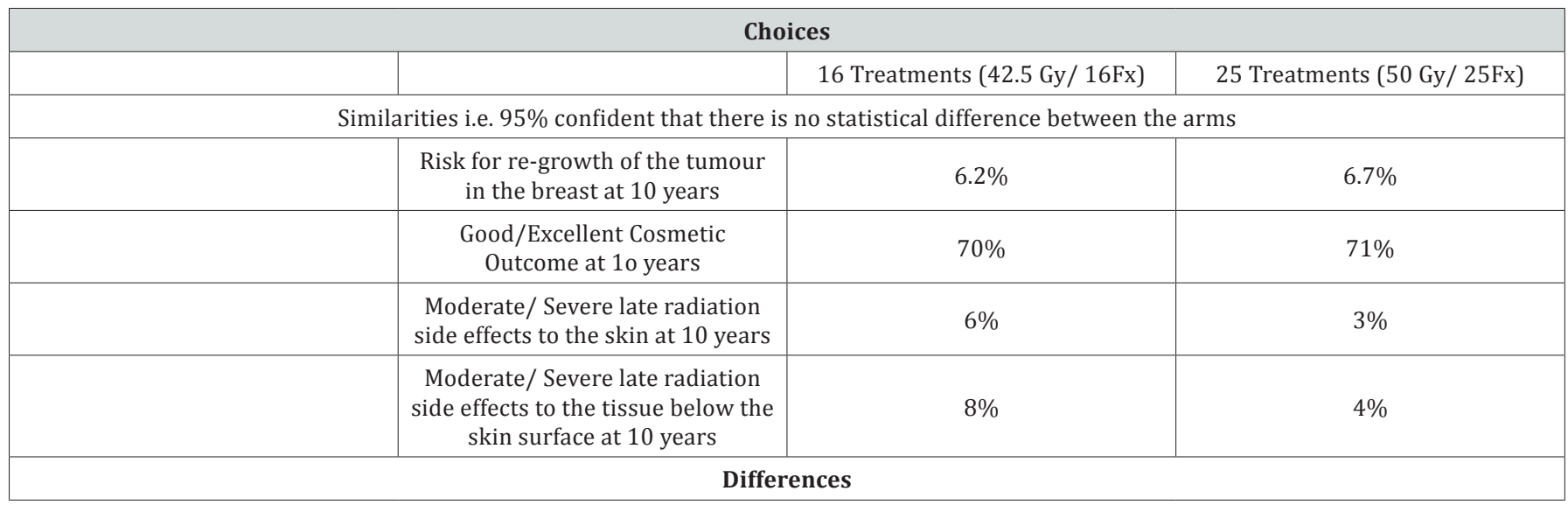




\section{Cancer Therapy \& Oncology International Journal}

\begin{tabular}{|c|c|c|c|}
\hline 1. & Median reported clinical follow up & 12 years & $>20$ years \\
\hline 2. & $\begin{array}{l}\text { Estimated cost to patient in } \\
\text { Singapore dollars }\end{array}$ & & \\
\hline & Government subsidized total cost & $\$ 2321$ & $\$ 2849$ \\
\hline & Private Singaporean total cost & $\$ 6392$ & $\$ 7898$ \\
\hline 3. & $\begin{array}{l}\text { Number of visits including } \\
\text { radiotherapy planning visit }\end{array}$ & 17 & 26 \\
\hline \multicolumn{4}{|c|}{ Reasons for your decision } \\
\hline \multicolumn{4}{|c|}{ Lower cost } \\
\hline \multicolumn{4}{|c|}{ More Convenient } \\
\hline \multicolumn{4}{|c|}{ Longer median follow up } \\
\hline
\end{tabular}

Based on one large previous medical study, there are to options for giving radiotherapy (RT) to your breast. We can treat with either 16 treatments (Fx) or 25 treatments (Fx). The treatments both have the same chance of controlling the disease in your breast - overall about $93 \%$ at 10 years from diagnosis. There is no difference in survival or quality of life between the treatments. However as the median follow up for the study is only 12 years, we cannot be certain that the results of the 2 treatments will remain similar beyond the first 12 years (Table 1).

Prior to commencement of this study, a pilot study of 10 healthy women volunteers (aged between 24-69 years old) was done to confirm instrument validity and reproducibility. Our pilot study found that $50 \%$ of patients chose HBRT. Thus the sample size was determined on a point estimate of $50 \%$ choosing HBRT, with $95 \%$ confidence interval from $40 \%$ to $60 \%$. A total of 74 patients were required

The study protocol was reviewed and received institutional ethics board approval. The study population was drawn from patients consulted by radiation oncologists at the National University Cancer Institute, Singapore. Eligibility criteria was similar to that used in the trial by Whelan et al. [8], i.e. women with staged $\mathrm{T} 1$ or $\mathrm{T} 2$ primaries, node negative adenocarcinoma of the breast cancer treated with lumpectomy and either sentinel lymph node biopsy or axillary clearance, maximum width of breast tissue $<25 \mathrm{~cm}$ and capacity to provide informed consent and take part in the process of shared decision making.

All potentially eligible patients were identified by participating radiation oncologists at the time of first consultation for radiotherapy. Using the decision board instrument as a visual aid, consenting eligible patients were counselled regarding the comparative risks and benefits of SBRT and HBRT. Patients and any accompanying family were allowed time to ask questions and clarify information. The choice of adjuvant radiotherapy fractionation was made either at that visit or confirmed at the time of radiotherapy simulation. After the decision was made, patients were asked without prompting, reasons behind their decisions. According to their decision, patients then received either whole breast irradiation of 42.5 Grays in 16 fractions over 22 days or whole breast irradiation 50 Grays in 25 fractions over
35 days. A boost to the tumour bed of 10 Grays in 5 fractions was used for all patients.

\section{Statistical Analysis}

The main outcome measure was choice of HBRT, and we used the binary logistic regression model to look at the association with the following factors: patient age, sex, race, education level, distance from home to hospital, travel time to hospital, mode of transport to hospital, paying class, employment status, social support, whether decision was made by the patient or family, stage of disease and performance status as scored by the Eastern Cooperative Oncology Group (ECOG).

Data analysis was performed using Stata V10.2 (Stata Corp, College Station, TX, USA), with level of significance set at $5 \%$.

\section{Results}

Patients were recruited over a 4 month period from November 2009 to March 2010. 74 consecutive patients with node negative stage I or II breast cancer were eligible and entered onto the study. None of the identified eligible patients refused participation in the study. The median age of consenting patients was 54 years old (range 36 to 72 years). $85.1 \%$ were Chinese, $5.4 \%$ were Malay and $6.7 \%$ were Indian. $77 \%$ of patients had high school education or greater and $45.9 \%$ were employed outside the home. $67.6 \%$ of patients received government subsidy for their treatment. $17.6 \%$ lived alone, $66.2 \%$ with spouse only and $16.2 \%$ with children. $78 \%$ of patients made the decision for which fractionation they wished to undergo on their own. Median distance from the patient's home to hospital was $9 \mathrm{~km}$ (range, 2 to $23 \mathrm{~km}$ ), median travel time was $20 \mathrm{~min}$ (range 5 to $60 \mathrm{~min}$ ) and $58 \%$ of patients used public transportation to reach the hospital.

Patient choice of fractionation schedule by characteristic is presented in Table 2. 36 of the 74 patients (48.7\%) chose the schedule of $42.5 \mathrm{~Gy}$ in 16 fractions (95\% confidence interval, $36.9 \%$ to $60.6 \%$ ). On univariate analysis, the only patient factor that predicted for patient's choice was increasing age (OR 1.09, $95 \%$ CI 1.03 to $1.15, \mathrm{p}=0.002$ ). Patients aged 50 years to 60 years were 5.42 times ( $95 \%$ CI 1.72 to $17.02, \mathrm{p}=0.004)$ and patients 60 70 years were 10.59 times ( $95 \%$ CI 2.4 to 46.75 , $\mathrm{p}=0.002$ ) more 


\section{Cancer Therapy \& Oncology International Journal}

likely to choose HBRT than patients less than 50 yrs. Patient decision-making was not analyzed to be significantly affected by any other patient factor ( $\mathrm{p}>0.1$ for all other variables). Patient Table 2: Patient choice of fractionation schedule by characteristic. decision-making was also not influenced by which radiation oncologist they consulted with (Table 2).

\begin{tabular}{|c|c|c|c|}
\hline Characteristic & $\begin{array}{l}\text { Number Choosing Hypo- } \\
\text { fractionation }\end{array}$ & $\begin{array}{l}\text { Number Choosing Standard } \\
\text { Fracationation }\end{array}$ & $\begin{array}{l}\text { Percentage Choosing Hypo- } \\
\text { fracationation (\%) }\end{array}$ \\
\hline \multicolumn{4}{|c|}{ Age (years) } \\
\hline$<50$ & 9 & 26 & $25.7^{*}$ \\
\hline $50-60$ & 16 & 7 & $69.6^{*}$ \\
\hline$>60$ & 11 & 5 & $68.8^{*}$ \\
\hline \multicolumn{4}{|c|}{ Race } \\
\hline Chinese & 29 & 34 & 46.0 \\
\hline Malay & 4 & 2 & 66.7 \\
\hline Indian & 3 & 2 & 60.0 \\
\hline \multicolumn{4}{|c|}{ Education level } \\
\hline Did not complete high school & 11 & 7 & 61.1 \\
\hline High school diploma and above & 25 & 31 & 44.6 \\
\hline \multicolumn{4}{|l|}{ Employment status } \\
\hline Unemployed/ Retired & 19 & 21 & 47.5 \\
\hline Part time/ Full time & 17 & 17 & 50.0 \\
\hline \multicolumn{4}{|c|}{ Cost } \\
\hline Government subsidized & 27 & 23 & 54.0 \\
\hline Private & 9 & 15 & 37.5 \\
\hline \multicolumn{4}{|c|}{ Social Support } \\
\hline Stays alone & 6 & 7 & 46.2 \\
\hline With spouse only & 22 & 27 & 44.8 \\
\hline With extended family & 8 & 4 & 66.7 \\
\hline \multicolumn{4}{|c|}{ Decision made by } \\
\hline Patient alone & 29 & 29 & 50.0 \\
\hline Jointly with family & 7 & 9 & 43.7 \\
\hline \multicolumn{4}{|c|}{ Distance from home/work place to hospital (km) } \\
\hline $2-9$ & 18 & 24 & 42.9 \\
\hline $9-23$ & 18 & 14 & 56.2 \\
\hline \multicolumn{4}{|c|}{ Travel time to hospital (min) } \\
\hline$<20$ & 18 & 22 & 45.0 \\
\hline $20-40$ & 11 & 8 & 57.9 \\
\hline $41-60$ & 7 & 8 & 46.7 \\
\hline \multicolumn{4}{|c|}{ Mode of transport } \\
\hline Public transport & 18 & 20 & 47.4 \\
\hline
\end{tabular}

Patients who chose HBRT did so for reasons of lower cost alone in $3.0 \%$, increased convenience alone in $58.3 \%$, and both lower cost and increased convenience in $38.9 \%$. The patients who chose SBRT cited factors that shaped their decision to be those of more clinical experience with its use and the longer length of follow-up available.

\section{Discussion}

Our study is the first to directly explore the question: "If the medical community assesses clinical equivalence between
HBRT and SBRT in terms of toxicity, local control and survival, when presented with the choice what would patients choose based on other end points such as cost, convenience and clinical experience?" The main findings in the current study were two-fold: First, that the majority of women with breast cancer preferred to be involved in the decision making process of radiotherapy regimen for their breast cancer; Second, that patient's perspective of risk differs from that of the physician on their behalf as shown by the difference in the age group of patients who were willing to accept HBRT over SBRT. Older 


\section{Cancer Therapy \& Oncology International Journal}

patients tended to choose HBRT, while younger patients were concerned about relative demonstrated duration of efficacy and preferred SBRT.

While older patients (OR 5.42 for patients 50-60 years of age and OR 10.59 for patients 60-70 years, compared to patients $<50$ years of age,) were more likely to choose HBRT for reasons of cost and convenience, younger patients (less than 50 years of age) placed greater weight on the length of clinical experience and follow up available. This difference in belief was reflected in the significantly higher number of women less than 50 years of age choosing SBRT over HBRT ( $p=0.002$ ) in spite of the considerable difference in financial cost borne by the patient between the two fractionation regimens (up to \$1506 Singapore dollars, approximately $\$ 1000$ US dollars) and added inconvenience of SBRT.

Adjuvant radiotherapy for early stage breast cancer is unique in various ways:

i. the heterogeneous patient group it serves. Patients in this group span a spectrum in terms of age, social commitments, and opinions on importance of factors such as survival, cosmesis and convenience.

ii. in the relative diversity of treatment regimens available with comparable outcomes. Treatment regimens that have been substantiated by long term evidence with 10 to 15 year followup exist for various courses of HBRT and SBRT. Recent evidence suggests HBRT may have a superior acute toxicity profile $[6,7]$. However the natural history of early stage breast cancer preferably would have duration of follow-up of more than 10 years for sufficient assessment of its efficacy.

iii. the share of a given Radiation Oncology department's clinical service that it occupies.

Length of follow up may still be of concern to patients, though medical opinion may generally interpret the existing published data to show both options equivalent by local control and survival. ASTRO guidelines from 2011 also highlight concerns with hypo fractionation in adjuvant breast irradiation as the single standard [24]. Despite promising START B data, concerns on the adoption of hypo fractionation in adjuvant breast irradiation include:

a) small representation of patients with regional lymphatic irradiation, hence generalizability of safety data from START trial to such patients

b) The risk of brachial plexus morbidity though likely low is unknown. Cardiac late toxicity in hypo fractionation compared with standard fractionation is also a concern.

c) An exclusion criterion of the START trials was immediate breast reconstruction. As immediate reconstruction is common practice in some populations including this study's, the use of START toxicity data may not apply here
These results serve as a useful reminder of the nuances of physician interpretation of current evidence. Furthermore, in this study, a script was not drafted for the consult- use of which would have been impractical- so presentation of the efficacy and toxicity profile of treatments would have understandably varied from consult to consult. While these results indicate the considerations important to patients when making a decision on adjuvant breast radiation therapy, it is noted that our trial was confined to a single institution with its unique parameters for value-driven healthcare. Singapore is a highly urbanized city state. No patient in our sample needed to travel more than 60 minutes for her daily treatment. In addition, the cost structure of radiotherapy as defined in our study may not be applicable to other countries. Our sample size consisted only of Asian patients whose views on treatment risk and whose weight age placed on different patient and treatment factors may be different from Western counter parts.

The decision board presented medical factors (survival and local control outcomes, late toxicities, duration of follow-up) generalizable across populations with different geographic and socioeconomic characteristics. But it also contained information that is unique to each patient population, in this case, convenience (in numbers of visits) and financial cost. An interesting area for future research would be to determine if convenience and cost, variables that would change in a different population setting, would be considered important enough for patients to decide differently, given the same medical information.

Patient preference between either adjuvant breast radiotherapy regimen is dependent on many factors, the importance of each being weighted differently according to the individual. Furthermore, aggregate preferences across populations with different cultural and social backgrounds would differ as well. Our study summarizes how these factors are considered and affect patients in a specific urban population in the context of the cost structure unique to Singapore during the period of the study's conduct. Future studies with a more diverse population from a broader geographic region, would provide a more rounded understanding of the impact of social factors and patients' opinion on medical outcomes, on decision-making, and may improve the generalizability of the results of this study. As breast radiotherapy comprises a large share of the workload for many centres especially in developed countries, such results will help in resource allocation and workforce projections

The results of our study illustrate the importance of considering individual patient preferences during the treatment decision making process. Thus it is clear that research into patient preferences for breast radiotherapy would inform organization of radiotherapy and inform healthcare policy and resource allocation. Our results offer insight into the discrepancy between medical opinion and patients' actual preference, and while informative and locally applicable, individual centres would benefit from local, centre-specific data to guide planning 


\section{Cancer Therapy \& Oncology International Journal}

at the level of an institution's Radiation Oncology unit and that of national healthcare planning.

\section{Conclusion}

Older patients chose HBRT for the advantage of cost and convenience, while younger patients chose SBRT because of longer length of clinical experience and follow-up. Research into patient preferences for breast radiotherapy would inform management of patient care and inform healthcare policy and resource allocation.

\section{Reference}

1. James ML, Lehman M, Hider PN, Jeffery M, Hickey BE, et al. (2010) Fraction size in radiation treatment for breast conservation in early breast cancer. Cochrane Database Syst Rev 16(3): CD003860.

2. Ribeiro GG, Dunn G, Swindell R, Harris M, Banerjee SS (1990) Conservation of the breast using two different radiotherapy techniques: Interim report of a clinical trial. Clin Oncol (R Coll Radiol) 2(1): 27-34.

3. Polgár C, Sulyok Z, Fodor J, Orosz Z, Major T, et al. (2002) Sole brachytherapy of the tumor bed after conservative surgery for T1breast cancer: Five-year results of a phase I-II study and initia findings of a randomized phase III trial. J Surg Oncol 80(3): 121-128.

4. Holmes DR, Baum M, Joseph D (2007) The TARGIT trial: Targeted intraoperative radiation therapy versus conventional postoperative whole-breast radiotherapy after breast-conserving surgery for the management of early-stage invasive breast cancer (a trial update). Am J Surg 194(4): 507-510

5. Dodwell DJ, Dyker K, Brown J, Hawkins K, Cohen D, et al. (2005) A randomised study of whole-breast vs tumour-bed irradiation after local excision and axillary dissection for early breast cancer. Clin Oncol (R Coll Radiol) 17(8): 618-622.

6. Jagsi R, Griffith KA, Boike TP, Walker E, Nurushev T, et al. (2015) Differences in the Acute Toxic Effects of Breast Radiotherapy by Fractionation Schedule Comparative Analysis of Physician-Assessed and Patient-Reported Outcomes in a Large Multicenter Cohort. JAMA Oncol 1(7): 918-930.

7. Shaitelman SF, Schlembach PJ, Azru I, Ballo M, Bloom ES, et al. (2015) Acute and Short-term Toxic Effects of Conventionally Fractionated vsHypofractionated Whole-Breast Irradiation: A Randomized Clinical Trial. JAMA Oncol Oct 1(7): 931-941.

8. Whelan TJ, Pignol JP, Levine MN, Julian JA, MacKenzie R, et al. (2010) Long-Term Results of Hypofractionated Radiation Therapy for Breast Cancer. N Engl J Med 362(6): 513-520.

9. Haviland JS, Owen JR, Dewar JA, Rajiv K Agrawal, Jane Barrett, et al. 92013) The UK Standardisation of Breast Radiotherapy (START) trials of radiotherapy hypofractionation for treatment of early breast cancer: 10 -year follow-up results of two randomized controlled trials. Lancet Oncol 14(11): 1086-1094.

10. Early Breast Cancer Trialists' Collaborative Group (EBCTCG), Darby S, McGale P, Correa C, Taylor C, et al. (2011) Effect of radiotherapy after breast-conserving surgery on 10-year recurrence and 15-year breast cancer death: meta-analysis of individual patient data for 10,801 women in 17 randomised trials. Lancet 378(9804): 1707-1716.

11. Dayes IS, Whelan TJ, Julian JA (2006) Cross-border referral for early breast cancer: an analysis of radiation fractionation patterns. Curr Oncol 13(4): 124-129.

12. Page BR, Belnap T, Bowen RC, Gaffney DK, Sause WT (2013) Utilization of hypofractionated and conventional breast radiotherapy in the State of Utah. Cancer and Clinical Oncology 2(2): 34-41.

13. Bekelman JE, Sylwestrzak G, Barron J, Liu J, Epstein AJ, et al. (2014) Uptake and Costs of Hypofractionatedvs Conventional Whole Breast Irradiation After Breast Conserving Surgery in the United States, 20082013. JAMA 312(23): 2542-2550.

14. Ashworth A, Kong W, Whelan T, Mackillop W (2013) A populationbased study of the fractionation of postlumpectomy breast radiation therapy. Int J Radiat Oncol Biol Phys 86(1): 51-57.

15. Whelan T, Marcellus D, Clark R, Levine M (1993) Adjuvant radiotherapy for early breast cancer: patterns of practice in Ontario. CMAJ 149(9): 1273-1277.

16. Tariman JD, Doorenbos A, Schepp KG, Becker PS, Berry DL (2014) Patient, physician and contextual factors are influential in the treatment decision making of older adults newly diagnosed with symptomatic myeloma. Cancer Treat Commun 2(2-3): 34-47.

17. Street RL, Haidet P (2011) How Well Do Doctors Know their Patients? Factors Affecting Physician Understanding of Patients' Health Beliefs. Gen Intern Med 26(1): 21-27.

18. Degner LF, Kristjanson LJ, Bowman D, Sloan JA, Carriere KC, et al. (1997) Information Needs and Decisional Preferences in Women With Breast Cancer. JAMA 277(18): 1485-1492.

19. Bruera E, Willey JS, Palmer JL, Rosales M (2002) Treatment Decisions for Breast Carcinoma: Patient Preferences and Physician Perceptions. Cancer 94(7): 2076-2080.

20. Hawley ST, Lantz PM, Janz NK (2007) Factors Associated with Patient Involvement in Surgical Treatment Decision making for Breast Cancer. Patient Educ Couns 65(3): 387-395.

21. Whelan TJ, Levine MN, Gafni A, Lukka H, Mohide EA, et al. (1995) Breast irradiation postlumpectomy: development and evaluation of a decision instrument. J Clin Oncol 13(4): 847-853.

22. Whelan T, Levine M, Gafni A, Sanders K, Willan A, et al. (1999) Mastectomy or lumpectomy? Helping Women Make Informed Choices. J Clin Oncol 17(6): 1727-1735

23. Shakespeare TP, Lu JJ, Back MF, Liang S, Mukerjee RK, et al. (2003) Patient preference for radiotherapy fractionation schedule in the palliation of painful bone metastases. J Clin Oncol 21(11): 2156-2162.

24. Smith BD, Bentzen SM, Correa CR, Hahn CA, Hardenbergh PH, et al. (2011) Fractionation for whole breast irradiation: asn American Society for Radiation Oncology (ASTRO) evidence-baded guideline. Int J Radiat Oncol Biol Phys 81: 59-68. 


\section{Your next submission with Juniper Publishers} will reach you the below assets

- Quality Editorial service

- Swift Peer Review

- Reprints availability

- E-prints Service

- Manuscript Podcast for convenient understanding

- Global attainment for your research

- Manuscript accessibility in different formats

( Pdf, E-pub, Full Text, Audio)

- Unceasing customer service

Track the below URL for one-step submission https://juniperpublishers.com/online-submission.php 